\title{
L'inspiration de la matière
}

Entretien réalisé par Aurélie Coulon

\section{Camille Boitel et Aurélie Coulon}

\section{(2) OpenEdition}

Journals

Édition électronique

URL : https://journals.openedition.org/agon/2076

DOI : $10.4000 /$ agon.2076

ISSN : 1961-8581

Éditeur

Association Agôn

Référence électronique

Camille Boitel et Aurélie Coulon, «L'inspiration de la matière », Agôn [En ligne], 4 | 2011, mis en ligne le 27 décembre 2011, consulté le 28 juin 2022. URL : http://journals.openedition.org/agon/2076 ; DOI : https://doi.org/10.4000/agon.2076

Ce document a été généré automatiquement le 29 septembre 2020.

Association Agôn et les auteurs des articles 


\title{
L'inspiration de la matière
}

\author{
Entretien réalisé par Aurélie Coulon \\ Camille Boitel et Aurélie Coulon
}

\section{NOTE DE L'ÉDITEUR}

Entretien réalisé le 27 mars 2011 à Grenoble

1 L'Immédiat a été créé le 13 octobre 2009 au Théâtre du Merlan, à Marseille. Ce projet mêle théâtre physique, performance et cirque. Sur le plateau, une accumulation d'objets récupérés, ramassés au fil de la création : «Les objets sont ceux du quotidien, disons simplement qu'ils ont dû s'adapter à de nouveaux besoins. Il s'agit plutôt de ceux dont personne ne voulait plus : les boiteux, les penchés, les fragiles, les tordus, les bancals, les troués, les cassés, les morcelés ${ }^{1}{ }^{\prime}$. Des personnages évoluent dans ce monde dévasté où tout s'écroule, et de déséquilibre en déséquilibre se construit une trame $\mathrm{d}^{\text {'accidents }}{ }^{2}$. Les interprètes se laissent surprendre par cet ensemble instable, et réagissent dans l'instant, dans cet immédiat que le spectacle cherche à saisir. Au cours du travail, une technique corporelle particulière s'est inventée, à travers la confrontation avec ces objets instables et l'expérimentation du déséquilibre. Puis, une écriture scénique a émergé des premières improvisations, sur le mode du bricolage. Entre maîtrise et improvisation, les situations se succèdent: les personnages sont menacés, se dissimulent, se démènent. Une table qu'on croyait solide s'effondre brusquement, entraînant une série de réactions en chaîne : peu à peu, tous les objets se brisent, se désarticulent. Ainsi, les objets permettent aux interprètes d'«improviser avec ce qui survient $»^{3}$.

2 Camille Boitel, formé auprès d'Annie Fratellini, a participé en tant qu'interprète à La Symphonie du Hanneton de James Thierrée. Lauréat de la première session des «Jeunes Talents Cirque ", il a créé la compagnie Lamereboitel en 2002. Avant L'Immédiat, il a créé en 2003 L'Homme d'Hus, spectacle dans lequel les objets - des tréteaux - sont aussi en scène. 


\title{
Les objets, à l'origine d'une manière d'être en scène
}

\author{
Aurélie coulon. Depuis quand s'est développé ton intérêt pour les objets au sein de tes \\ spectacles? Comment s'élabore la création artistique à partir des objets, que ce soit pour \\ ton dernier spectacle, L'Immédiat', ou pour tes créations plus anciennes?
}

Camille Bortel. J'ai commencé à faire des spectacles avec des objets parce qu'ils me permettaient de considérer différemment le fait d'être sur scène. Le plaisir que j'ai pris à être sur scène était dès l'origine lié aux objets, parce qu'ils me permettaient d'être en retrait par rapport à la présence première qu'on peut avoir sur un plateau. J'ai eu le sentiment de faire vivre les objets, mais pas au sens marionnettique du terme, c'est-à-dire non pas en leur donnant la vie, mais en leur donnant plutôt des sensations, dans leur matière même, en créant des effets à partir de la matière de l'objet. Ce rapport particulier aux objets m'a donné envie d'aller sur scène parce qu'il déplaçait totalement mon rapport à la scène, ma façon de me placer, d'entrer et de jouer sur le plateau. Je me sentais en quelque sorte mieux placé, au sens où j'étais au service de quelque chose et non plus dans une perspective de représentation où il aurait fallu donner quelque chose de moi, de ma personne en jouant avec ma propre image. Lorsque je construis quelque chose avec les objets, je suis tout de suite dans le concret de la matière, ce qui me permet de ne pas me poser la question de mon existence en scène, d'être non plus l'acteur mais le porteur des choses qui se mettent en place progressivement sur le plateau. Ce rapport particulier à la scène est important pour moi au sens où j'ai trouvé par le biais de la manipulation d'objets, la meilleure manière d'être littéralement placé sur la scène.

Ce que j'aime retrouver aussi dans les spectacles de marionnettes, c'est cette différence de placement : j'aime voir le manipulateur de la marionnette, sa présence, sa façon d'apparaître, de se placer ou de s'effacer, de se mettre au service de la chose, d'être concentré face à l'objet. C'est le placement du manipulateur qui me parle, beaucoup plus que la marionnette elle-même. Parce que la manipulation te met nécessairement dans une tension, une concentration et une projection très particulières. Il y a toujours le développement de quelque chose qui a à voir avec le retrait dès l'instant qu'on travaille avec des objets. Les objets ne parlent pas depuis le vide, ils se chargent de signes avec la manipulation. Et certains objets amènent déjà leurs propres signes. Ce sont ces objets-là qui m'intéressent en priorité. Je n'ai pas choisi de travailler avec des objets appartenant aux arts du cirque du type " massue » parce qu'ils n'ont pas pour moi de rapport direct à la vie quotidienne. Ce sont les objets que l'on connaît qui m'intéresse, comme une chaise ou une bouteille d'eau par exemple, c'est-à-dire des objets avec lesquels on a une sensation physique, des objets qui sont en contact avec la matière comme nous, et qui ont donc aussi une histoire. Un objet abîmé porte les signes de sa vie passée tout comme une personne possède des signes particuliers, des traits qui lui appartiennent en propre et définissent sa vie, toutes ces choses qui sont concentrées dans la notion de « regard». Les objets ont été usés par des hommes et des femmes; parce qu'ils sont au contact de l'humain, ils transportent le passé et créent des affects et des liens par leur présence. On est liés aux objets d'abord par la sensation, qu'elle soit de l'ordre de l'agréable ou du désagréable. 


\title{
Les objets dans L'Immédiat: de la collecte à la construction
}

\begin{abstract}
A.c. Justement, pourrais-tu revenir sur l'histoire des objets présents dans ton dernier spectacle, L'Immédiat? D'où viennent-ils? Dans un précédent entretien, tu nous avais expliqué que les objets du spectacle étaient ramassés sur les lieux de la représentation pour ensuite être abandonnés sur place, l'idée étant de refaire à chaque représentation un spectacle avec de nouveaux objets trouvés au hasard. Mais petit à petit, le spectacle s'est en quelque sorte fixé autour de plusieurs objets qui vous ont suivis lors de la tournée. Comment expliques-tu la réutilisation de certains objets et l'abandon des autres ? Pourquoi et comment s'effectue ce choix?
\end{abstract}

C.B. Ce spectacle est l'aboutissement d'une longue expérience. Effectivement, on a d'abord éprouvé le besoin de se servir, pour faire le spectacle, de tout ce qu'on trouvait sur place en ayant justement pour objectif de ne pas choisir, de ne pas trier les objets : on prenait des objets abîmés retrouvés dans la rue, parfois même des fragments d'objets et on demandait aux gens des objets dont ils ne se servaient plus. L'idée était vraiment de parcourir les rues des villes où avait lieu la représentation en ayant d'abord les mains vides pour ramasser progressivement les choses et les traîner derrière nous, à la manière de mendiants ou de collecteurs. On a commencé par partir en collecte sans transport, sans chariot ni roue, pour avoir l'occasion de trouver sur place les moyens de transporter les objets. On revenait ensuite avec de la matière qu'il fallait nettoyer et sélectionner. Certains objets sont alors apparus comme plus à même de raconter ce qu'on avait envie de dire à travers le spectacle. En s'appropriant les objets, en jouant avec, on pensait pouvoir au bout d'un certain temps acquérir une sorte de savoir-faire quant au choix des objets et leur manipulation. On pensait acquérir une forme d'expérience vive, mais en partant de rien, c'est-à-dire sans qu'il n'y ait aucun outil, aucun intermédiaire entre nous et les objets. C'est aussi dans ce but qu'on a d'abord refusé le moyen de transport.

$\mathrm{Au}$ fur et à mesure, cette aventure de collecte autour du spectacle prenait tant de place par rapport à celui du plateau, consacré à la création à proprement parler du spectacle lui-même, qu'on a eu l'impression que le spectacle était déjà là avant d'être créé : à travers les rencontres qu'on pouvait faire et la parole qu'on propageait à propos du spectacle, on a en quelque sorte engendré une forme de diffusion particulière d'un spectacle qui n'existait pas encore mais dont l'histoire était déjà faite. Cette expérience en amont du spectacle a alors considérablement réduit notre temps de travail sur le plateau, en déséquilibrant totalement le temps imparti à la collecte puis à la création du spectacle. Surtout, on a été confronté à un manque de maîtrise évident vis-à-vis des objets eux-mêmes. On pensait qu'on apprendrait vite à travailler avec cette matière mais l'expérience a montré que non : on apprenait très lentement, parfois même on régressait, si bien que le spectacle était vécu par nous comme un échec permanent, quelque chose de raté mais consciemment en quelque sorte car on savait que le spectacle était toujours inabouti. Cette conscience de l'échec faisait partie du processus qu'on avait décidé de suivre depuis le début.

La collecte des objets et leur abandon juste après le spectacle faisaient partie d'un processus plus large qui consistait à ne pas privilégier un objet plutôt qu'un autre et à refuser de s'attacher à des choses précises. Ces objets jetés, ramassés par nous pour ensuite être de nouveau jetés, avaient ainsi vécu une transformation assez forte dans la mesure où on les bricolait énormément. Au départ d'ailleurs, on voulait un 
spectacle construit à partir de fragments et non d'objets entiers, ce qui permettait de bricoler véritablement chaque morceau et de recomposer bout par bout l'intégralité des objets servant au spectacle, y compris jusqu'aux vêtements. Mais c'était un travail énorme qui empiétait trop sur le temps convenu pour le travail du plateau uniquement. Il a bien fallu renoncer à cette exigence de collecte car elle demandait une forme de systématisation qu'on n'était pas encore capable de mettre en place: cette idée de départ exigeait de nous d'être des Sisyphe capables de construire un système, une répétition systématique en dépit des aléas induits par la collecte des objets. C'est cette absence de maitrise vis-à-vis des objets que j'évoquais tout à l'heure qui nous a conduit à renoncer à la collecte telle qu'on la pratiquait au départ. Le spectacle s'est construit avec des personnes qui, pour beaucoup, en étaient à leur première expérience artistique vis-à-vis des objets ; certains ont décidé de continuer l'aventure, y compris en montant pour la première fois sur scène et c'est aussi à partir de cette inexpérience-là que le spectacle a vu le jour.

C'est dans ce même état d'esprit qu'on avait choisi de faire nous-mêmes la lumière et le son, d'être nos propres techniciens et machinistes, ce qui continuait de transformer la création de ce spectacle en une sorte d'aventure naufragée à l'avance. En France, on a joué dans deux ou trois théâtres mais on a rapidement renoncé car ce type de spectacle créait trop de tensions avec les personnes travaillant pour chaque lieu, chacun ayant peur de cette forme non aboutie et totalement close sur ellemême. Et c'est vrai qu'en y repensant, il y avait de quoi avoir quelques craintes...

A.c. Pourquoi ces craintes? Est-ce que la nature du spectacle entravait leur propre intervention? Est-ce parce que l'inconnu que chaque représentation pouvait signifier était contradictoire avec le caractère purement technique d'un spectacle devant se répéter chaque soir indépendamment du lieu?

C.B. Oui, effectivement. La création du spectacle a eu lieu au Théâtre du Merlan à Marseille, qui a soutenu notre projet depuis le début. On avait été accueillis en résidence dans un lieu qui dépendait du Merlan pendant toute la durée des travaux de rénovation du théatre, leur salle étant alors indisponible. Ils nous attendaient depuis longtemps et pour la réouverture du théâtre, on a présenté ce spectacle c'està-dire qu'on a collecté de nombreux objets en vue de remplir le théâtre alors qu'ils venaient justement de le vider. On avait transformé leur théâtre remis à neuf en une sorte d'usine de recyclage, ce qui a provoqué quelques incompréhensions. Il y avait des objets dans tous les sens, tout le monde était abasourdi. Cette représentation au Merlan a été incroyable car on n'avait fixé aucune limite surtout concernant le temps: on travaillait jusque quatre heures du matin sans jamais approcher l'idée d'une limite ou d'une fin possible à ce travail. Ça a donné un spectacle euphorique et déraisonnable, qu'on ne pouvait pas reproduire. Mais l'euphorie alternait toujours avec un sentiment d'errance totale parce qu'il arrivait qu'on ne sache plus quoi faire avec tous ces objets sur le plateau. Pour ce spectacle, les objets nous torturaient au sens où il fallait sans cesse les manipuler, y revenir, s'en occuper afin de pouvoir faire émerger quelque chose. Le spectacle était donc très irrégulier quant au rythme puisque se succédaient des moments d'errance où on ne savait plus quoi faire des objets et des moments de création intense. Tout était de l'ordre du bricolage comme si le spectacle s'était créé à partir de morceaux de cartons et d'un tas de projecteurs, c'est-à-dire quelque chose de très artisanal, conçu à partir du caractère épars de la matière. 
Quand certains techniciens ont vu ce qu'on était en train de construire, ils ont eu quelques craintes en dépit du soutien que nous apportait la direction du Théâtre. Nous étions dans deux mondes différents: eux qui avaient un savoir-faire qu'ils avaient appris et nous qui essayions de ne rien savoir faire à l'avance, de tout apprendre sur le tas. Et c'est ça qui représentait pour eux un vrai danger.

Mais ce qui est terrible, c'est que le spectacle tel qu'il existe aujourd'hui, c'est-à-dire dans une forme assez aboutie et stable, est beaucoup plus dangereux que dans sa forme initiale. La reproductibilité créé du danger au sens où elle instaure une forme de fatigue de la répétition qui peut être dangereuse et qui contraste avec la vigilance accrue et particulière qu'induit un spectacle unique.

$\mathrm{Au}$ fur et à mesure, le spectacle s'est néanmoins fixé autour d'objets qu'on jugeait adéquats : une armoire qui « jouait » bien, une lampe accrochée, des tables... C'est en expérimentant les objets au fil de nos découvertes qu'on a pu juger quels objets pouvaient rester ou non. Selon notre parcours, on essayait peu à peu de retrouver sur le lieu nouveau les objets qui avaient bien marché dans les représentations précédentes, des objets devenus précieux pour nous comme par exemple les gros manteaux qu'on avait trouvés à Vilnius et qu'on a décidé d'inclure définitivement dans le spectacle. On a finalement bâti une structure par rebond au fil des représentations, d'un lieu à un autre. A partir du foisonnement des choses expérimentées, on a gardé certaines scènes comme des souches à partir desquelles on réécrivait le spectacle en fonction du lieu dans lequel on se trouvait: le spectacle a ainsi eu plusieurs versions selon qu'il était joué en France ou à l'étranger, en Thaïlande par exemple où pendant trois mois, on a travaillé sur une version du spectacle qui prenait en compte, dans la manipulation des objets, l'art traditionnel du pays, ce qu'on ne pouvait pas garder pour les représentations en France. L'urgence du temps aussi a fait qu'on a gardé certains objets pour les représentations à venir : tout cela s'est pressé et même compressé pour obtenir peu à peu une forme stable. C'est par infusion que le spectacle s'est construit et non par choix délibéré ou par contrôle sur les objets. Au contraire, ce sont les objets qui se sont déposés tout seuls pour ainsi dire. Le fait que le spectacle n'ait pas été écrit lui confère une forme de fulgurance et une émotion particulière qui a justement à voir avec le fait que la création n'était pas due à un savoir-faire théâtral ou scénique particulier. C'est à partir des objets qu'il s'est construit et à partir d'eux en tant qu'objets, non en tant qu'accessoires de théâtre. Cette différence est importante. A la différence des accessoires conçus spécifiquement en vue du jeu et qui sont donc solides, résistants et fabriqués en vue de cette utilisation scénique, les objets qu'on a utilisés étaient fragiles et incommodes. C'était essentiel d'avoir ces objets réels sur le plateau. Je n'aime pas les actes faux en général et les objets ont cette particularité de ne jamais mentir: les sensations que procurent la lourdeur ou la légèreté d'un objet sont vraies. Ce poids a de l'importance parce qu'il induit une résistance ou une douleur vraie qui implique de la part de l'acteur de ne pas tricher : on souffre à porter soi-même un objet lourd et cela crée un investissement particulier, une conscience à part. Je pense que travailler avec de tels objets est d'abord uniquement désagréable et que seule la maîtrise progressive de l'objet permet d'éprouver ensuite le plaisir de la manipulation. 


\section{Corps et objets : impulsions, déséquilibres, situations}

A.c. La façon dont tu racontes le processus de création de ce spectacle me fait penser aux techniques du " contact improvisation », au sens où vous vous êtes servis d'une technique qui s'est inventée au fur et à mesure d'une pratique, par le contact avec les objets, en prenant en compte leur poids et les possibilités d'équilibre ou de déséquilibre qu'ils offrent. Une technique qui s'est adaptée constamment aux objets nouveaux, aux rencontres...

C.B. Travailler d'abord avec le poids de chaque objet, avec la force de gravité, nous a conduit à nous interroger sur la naissance du mouvement: d'où vient l'impulsion première? Quand on est spectateur, on ne sait pas forcément d'où provient cette impulsion et surtout si cette impulsion provient des objets eux-mêmes.

L'improvisation avec les objets est la partie de la création que je préfère parce que cela demande une vraie maîtrise de soi : l'objet ne ment pas, lorsqu'il vous fait mal, lorsqu'il se brise, lorsqu'il tombe, il le fait pour de vrai, il est radicalement dans le réel. Il incarne même le réel. C'est aussi en ce sens que je ne souhaite pas travailler sur un plateau nu. Pourtant, un plateau nu peut être magnifique : voir quelqu'un arriver les bras le long du corps dans un espace vide, c'est une messe très importante et très belle, qui puise justement sa force dans le fait qu'elle n'est pas dans le réel. Travailler avec les objets apporte au contraire ce réel très précieux pour mon travail : dans la vie, nous ne sommes jamais les bras le long du corps dans un espace vide; nous sommes toujours assis sur, accoudé à, appuyé contre. C'est ce rapport-là qui m'intéresse. Personnellement, je n'aime pas beaucoup les objets ; c'est le vivant plus que l'objet qui me plaît. Ce qui m'intéresse dans l'objet, c'est de voir celui qui se cache derrière et qui a inventé quelque chose avec cet objet, ce rapport vivant à la matière. Car les objets sont aussi une menace permanente pour moi, un peu à la manière d'une valise qu'on transporte en voyage et qui nous encombre parce qu'on ne sait pas où la mettre ou qu'on a peur de la perdre : l'objet gâche alors le plaisir du voyage, il nous alourdit en même temps qu'il nous soutient.

Cette lourdeur n'est pas une chose agréable, y compris pour la création d'un spectacle. Je pense à l'expérience de Marine, danseuse sur ce spectacle, qui n'avait jamais travaillé avec des objets auparavant. Elle a dû s'approprier les objets, les apprivoiser, ce qui a demandé beaucoup de temps avant de pouvoir construire vraiment quelque chose : elle a commencé à apprécier les appuis que proposaient les objets au bout d'une période assez longue de découverte et de manipulation. Mais quand vient la maîtrise, alors s'ouvrent des perspectives de création immenses. L'addition d'un objet et d'une personne crée une situation. Celle-ci est nécessairement familière au spectateur car l'objet implique une forme de reconnaissance et une mémoire: le spectateur peut se projeter facilement sur la personne du manipulateur ou du danseur car il a déjà connu lui-même cette mise en situation à l'égard de l'objet. Le moindre geste est déjà connu de celui qui regarde et c'est l'objet qui induit cette reconnaissance immédiate. Par exemple, voir quelqu'un faire un saut périlleux peut faire frissonner, mais un spectateur qui n'a jamais pratiqué l'acrobatie n'est pas impliqué de la même manière que s'il voit quelqu'un tomber à la renverse: dans ce cas, le ressenti est différent car la mémoire est sollicitée, ce qui crée une reconnaissance de la situation. 
A.c. D'ailleurs, pendant le spectacle, la salle réagit énormément. Les gens rient, frissonnent, sursautent...

C.B. Oui, parce que les gens sentent que les objets ne trichent pas. Ils se rendent tout à fait compte de ce que ça pourrait faire d'être à la place de l'interprète, alors qu'un plateau nu déréalise beaucoup un spectacle. Les objets apportent beaucoup de réalité, avec toutes les difficultés que cela implique: les déplacements sont longs et deviennent vite monotones. Pour nous, c'est parfois décourageant en répétition de devoir déplacer tous les objets à chaque fois. Cela nous impose des contraintes.

Je parle des contraintes, mais c'est en travaillant avec les objets que j'ai eu le plus de plaisir à aller sur scène. Je me sentais au service de quelque chose, c'était la manière de travailler la plus juste pour moi. J'ai d'abord aimé jouer, improviser, puis, quand j'ai commencé à avoir envie d'écrire, c'est passé par les objets. La manipulation des objets peut amplifier nos gestes ou créer des espaces différents, mobiles. Quand on déplace un objet, on modifie l'espace. L'armoire blanche est une charnière dans le spectacle : quand on est devant, on a une présence complètement différente, cela crée un plan supplémentaire.

Improviser avec les objets, ce n'est vraiment pas une mince affaire. Je pense que les personnes qui ont participé à ce projet ont été très déroutées par ce travail, parce qu'elles avaient des expériences très différentes qui ne relevaient pas de la maîtrise artistique, c'est-à-dire de la capacité à considérer ce que le spectacle peut raconter, à être en jeu, tout en maîtrisant la technique et la matière. Une personne peut avoir un rapport complètement obsessionnel aux objets, et ressentir une certaine souffrance dans ce monde où les objets sont malmenés; une autre aura un rapport éthéré à l'artistique et sera toujours dans un rapport sensible aux objets, et une autre sera dans un rapport esthétique, à la recherche de la maîtrise. Chacun est un peu dérouté. Et puis les objets, quand on les prend sur les tibias, on ne peut pas ne pas les détester. Cela nous est arrivé souvent. Au début, on a travaillé pendant un an sans aucun objet. Pourtant, d'une certaine manière, les objets étaient déjà présents. Quand ils sont arrivés, cela a créé une sorte de choc, ils nous ont envahis, comme quand un appartement se trouve rempli par une accumulation de choses. On était envahis, et en même temps on n'avait pas envie de les lâcher. C'est cette contrainte qu'ils apportent qui est intéressante.

\section{Le bricolage comme écriture scénique}

A.c. Comment se passe l'écriture de l'objet dans ce spectacle? Au lieu d'être dans le narratif, on dirait que cette écriture crée tout de suite des impulsions, des situations et des actions. Est-ce que ce sont les objets qui permettent de créer des actions?

C.B. Oui, c'est ce qu'on cherche. On part plutôt de l'involontaire, même s'il y a évidemment beaucoup de passé, d'affectif et de sensations qui entrent en jeu. On ne peut pas totalement maîtriser ce qui se passe, le spectacle est comparable à des particules qui s'assemblent et se désassemblent. On n'a que des morceaux qu'on ne peut pas totaliser, à la différence d'une histoire qu'on pourrait résumer. C'est un spectacle impossible à résumer, parce que les choses arrivent et s'imposent à nous. Les objets concrétisent immédiatement ce qui se vit. Parce qu'il y a un objet, cela crée tout de suite une situation. Deux personnes l'une en face de l'autre, cela pourrait être le début d'une histoire, mais si elles poussent une armoire, c'est tout de suite une 
situation. Le simple fait d'avoir choisi des objets du quotidien nous transporte dans le quotidien.

Mon premier travail de ce type a consisté à détourner des objets de leur utilisation, mais ce n'est pas vraiment le cas ici. Pour L'Immédiat je ne pense pas pouvoir signer l'écriture du spectacle, je dirais plutôt que le spectacle est ce qui nous est arrivé à partir d'un projet d'écriture. On a tous été embarqués, c'est comme un naufrage, et je suis très content qu'on ait tous fait ce naufrage ensemble. Les objets nous ont obligés à concrétiser très vite l'écriture du spectacle : on les met à tel endroit, ça ne marche pas, alors il faut les déplacer, et ainsi de suite. Le spectacle se compose d'une série de constats d'échec. D'ailleurs, pendant la deuxième partie, on sent que ça va se terminer parce que tout est encombré. Tout est fini et on continue quand même, alors que l'espace est saturé. Les objets rendent les actes immédiats, et cela nous a très vite obligé à de la précision dans l'écriture, ne serait-ce que pour gérer le danger amené par les objets, pour ne pas prendre quelque chose sur la tête. C'est un danger à distinguer du risque que l'on court lorsqu'on fait quatre sauts périlleux au-dessus du vide, dans le cas de la prouesse technique, mais ce danger est réel. L'accident et l'objet sont très liés, en fin de compte. L'accident d'un corps face à un autre corps, c'est relativement mou et souple, alors qu'un corps face à un objet, c'est une confrontation plus radicale.

A.c. L'accumulation, le mouvement brouille la frontière entre le vivant et l'inerte dans le spectacle, cela crée parfois pour le spectateur une confusion entre le corps et l'objet.

C.B. L'objet est comme la concrétisation d'une impulsion. Dans le spectacle, ce qui tombe est forcément le résultat de quelque chose. Un simple objet nous donne déjà une conception du monde, et ces objets qui s'effondrent disent quelque chose du monde. Il y avait une table, on pensait que ça serait stable, et tout s'écroule. Beaucoup de choses nous échappent dans ce travail : je ne sais pas si ce sont des objets vivants, ou hantés. C'est surtout une déliquescence : ce sont des choses qui ne tiennent plus, et pourtant les gens continuent à vivre dans un monde qui ne tient plus du tout, et ne semblent pas particulièrement traumatisés.

A.C. Cela devrait mettre à mal l'illusion, et c'est l'inverse qui se produit. On a le sentiment que les objets sont vivants, qu'ils agissent, qu'ils menacent les interprètes.

C.B. Oui, c'est étonnant qu'on ait envie de projeter ainsi la vie sur les objets. En même temps, ils sont vivants à leur manière : ils sont usés, patinés, abîmés, ils ont une histoire. Il y a un trou dans l'armoire parce que quelqu'un a donné un coup de poing dedans, et si on avait décidé de le faire nous-mêmes le résultat ne serait pas du tout le même. Cette inspiration de la matière, qui raconte forcément des histoires qui nous échappent en partie, a été très importante dans le processus de création du spectacle. Aujourd'hui, je conserve le désir d'utiliser cette forme : avec des objets, en arrivant quelque part, on peut tout de suite créer quelque chose. Mais je crois que je m'y prendrais différemment. À cette étape du travail, il est difficile d'être dans un groupe avec des avis différents. Il faudrait créer une sorte d'écoute infime, être complètement dégagé de la nécessité d'expliciter certains choix. C'est comme de l'aïkido, mais avec des objets. En Thaillande, c'est moi qui nettoyais les objets collectés et je m'entraînais souvent avec. J'ai beaucoup travaillé avec des objets mis en série, des woks ou des tréteaux. Pour L'Homme d' Hus, j'avais cent vingt tréteaux. Ils me permettaient de déployer une multiplicité d'images, ils devenaient des mitraillettes, des troncs, des machines, des tas, des troupeaux d'animaux qui m'agressaient. Ce 
procédé avait beaucoup d'impact car le tréteau était un objet simple mis en série, qui pouvait se prêter à toutes sortes d'interprétations. Avec L'Immédiat, on est dans l'hétéroclite. En général, ce n'est pas ma sensibilité : je préfère travailler avec un élément très simple et faire des variations. Dans ce spectacle, on part de la périphérie et il n'y a pas de centre. L'Immédiat est dans l'interrogation, et non dans l'affirmation. Il y a des gens, dans le public, que ça dérange.

Pourtant, la tension narrative peut y être, même si on ne raconte pas une histoire. Ce spectacle est décousu, incontrôlable, et c'est ce que j'aime dans ce travail. Il ne ressemble jamais vraiment à ce qu'on aurait voulu faire. Mon dernier spectacle, L'Homme de Hus, était plus précis. Dans L'Immédiat, il y a une dilatation très particulière du temps. C'est vraiment lié à cette matière qu'on a choisi d'explorer. L'Immédiat, dès que tu veux le saisir, c'est fini. Les objets sont des sortes d'intermédiaires particuliers. Ils ralentissent les choses, ils donnent du temps, et parfois ils l'accélèrent car ils permettent de se cacher et de surgir, par exemple.

A.c. Est-ce que ces objets sont des éléments rythmiques?

C.B. Oui, dans le spectacle, ils ont des fonctions rythmiques, sonores, visuelles et scénographiques.

A.c. Est-ce qu'il n'y a pas tout de même un objet fabriqué dans L'Immédiat? Je pense à la machine qui se trouve à jardin. Qui l'a construite?

C.B. À la toute fin, nous avons fabriqué quelques objets pour pouvoir «tourner » plus facilement. C'est le cas des accroches qui nous permettent de manipuler les pendrillons. Mais en fait, la machine ne s'intègre pas bien au spectacle, on est tout le temps en train de se demander si on ne va pas l'enlever. Nous l'avons fabriquée nousmêmes, avec deux autres personnes qui sont venues nous aider de temps en temps. Nous avons récupéré beaucoup de choses: les pendrillons, une table d'écolier, de la ferraille. Nous avons été accompagnés par un excellent constructeur, qui est arrivé à la fin. C'est quelqu'un d'incroyable, qui est dans la maîtrise, dans l'organisation. Il nous a fait une cuisine de tournée entièrement équipée, récemment, il a fait un petit bateau, il a aussi construit son atelier de travail. «Constructeur » est un terme un peu maigre : c'est quelqu'un qui travaille la matière. Il est en train de partir, après un an et demi de collaboration. Il se fait remplacer car ça devenait un peu trop dur pour lui de travailler avec nous, parce que le spectacle bouge tout le temps, et ça le faisait souffrir de voir cet ensemble instable, ces objets pourris. Il a réussi à construire quelques objets qui nous ont bien servi : cette machine, une sorte de catapulte avec une petite main qui dépasse, et quelques éléments pour accélérer le montage et le démontage. Mais notre mode de fonctionnement complètement désorganisé n'était pas adapté pour lui, ce n'était pas le lieu pour un constructeur. On ne lui laissait pas vraiment la place d'apporter des améliorations : si on change les objets, ce n'est plus du tout la même histoire. Si on refaisait le spectacle avec des objets construits, je ne pense pas que ça donnerait la même sensation. Le spectacle serait plus rythmé, plus surprenant, mais les situations ne seraient pas les mêmes.

A.c. Ce que tu dis des objets pourrait s'appliquer au spectacle lui-même : il est précis, mais il n'est pas construit.

C.B. On a réparé et consolidé les objets, tout le monde a participé, y compris la danseuse. J'aime beaucoup construire les choses sans savoir-faire, trouver des solutions assez astucieuses pour que ça marche: ne pas avoir d'atelier, ni d'outils. 
C'est le mode de composition de L'Immédiat. C'est très efficace et agréable de travailler dans un atelier, mais chaque œuvre a son mode de composition, et on ne peut pas lui transposer d'autres méthodes. Dans L'Immédiat, la texture de ces objets et de ces corps est importante. Une danseuse qui n'a jamais porté des choses lourdes aura un contact beaucoup plus doux avec les objets que des acrobates, par exemple. Ces objets sont aussi le reflet de l'écriture, qui est une sorte de bricolage, quelque chose de débraillé, qui tombe en ruines. L'écriture ne cesse pas de changer, on a l'impression qu'elle ne cesse pas de se dégrader, même si c'est mieux qu'avant. Il y a un désespoir réel face à cette résistance du matériau. Il n'y a pas longtemps, j'ai joué sans aucun objet, et je me suis senti dans une joie incroyable. Mais en fait, il y avait tout de même une table, une chaise, une petite robe, une perruque. Dans ce cas-là, les objets sont des appuis de jeu au sens plus traditionnel du terme.

A.c. Ta démarche, et en particulier les moments de collecte qui ont précédé les premières représentations, me fait beaucoup penser à la démarche décrite par Agnès Varda dans Les Glaneurs et la glaneuse. Est-ce que cette idée de glanage est présente dans ton travail ?

C.B. Pour moi, à l'origine de ce travail il y avait une démarche esthétique qui était très liée à la définition que Claude Lévi-Strauss donne du bricolage dans La Pensée sauvage. On accumule des fragments, et très vite, c'est la matière elle-même qui te donne des idées. C'est aussi valable pour le langage : une fraise, ça peut être un fruit, mais aussi un outil pour percer. Le bricolage, c'est une manière d'être influencé par la matière. Le spectacle est presque écrit par la matière, par les hasards et en même temps il y a la rigueur d'une vitalité à continuer: cela fait des années qu'on travaille sur ce spectacle, il y a une persévérance, un acharnement moteur, l'illusion que quelque chose va se passer. L'illusion, parce que depuis qu'on tourne, on a joué soixante-dixhuit fois, et à chaque fois ce qui se passe n'est pas tout à fait ce qu'on attendait. Ce n'est pas le temps du spectacle le plus important, il reste un ensemble encore plus fort, qui est ce qu'on a vécu dans l'aventure. Parmi les gens qui sont là depuis le début, il y a encore des gens qui sont nostalgiques de l'époque où on était sans objets. $\mathrm{Tu}$ marches et tu ramasses quelque chose qui te fait penser à autre chose, tout communique, les influences sont multiples: ce n'est pas toi qui t'arrêtes et qui décides d'écrire ceci. Quand on écrit, on peut être clinique ou bricoleur. Ce chapitrelà de mon travail correspond plutôt au bricolage.

\section{NOTES}

1. Présentation du spectacle, programme de salle de la MC2 - Maison de la culture de Grenoble. 2. À ce sujet, voir "Un partage de déséquilibres », entretien réalisé par Alice Carré et Barbara Métais-Chastanier pour le dossier «L'accident »: https://journals.openedition.org/agon/1268 [consulté le 25 décembre 2011].

3. La formule a été utilisée par Camille Boitel dans l'entretien « Un partage de déséquilibres ».

4. L'Immédiat, spectacle conçu par Camille Boitel, se joue à Toulouse au Théâtre Garonne du 14 au 21 décembre 2011, puis sera repris les 9 et 10 février 2012 à la Scène nationale d'Orléans. Avec la participation de Marine Broise, Aldo Thomas, Camille Boitel, Pascal Le Corre, Jérémie Garry (en 
alternance avec Thomas Debroissia et Marion Lefebvre), Jacques-Benoît Dardant. Production et diffusion: Si par hasard.

INDEX

Mots-clés : Boitel (Camille), objet 